\title{
$\mathrm{BFHA}$ \\ (A)
}

BETTER FUTURE of HEALTHY AGEING 2020

\section{Students' Posters}

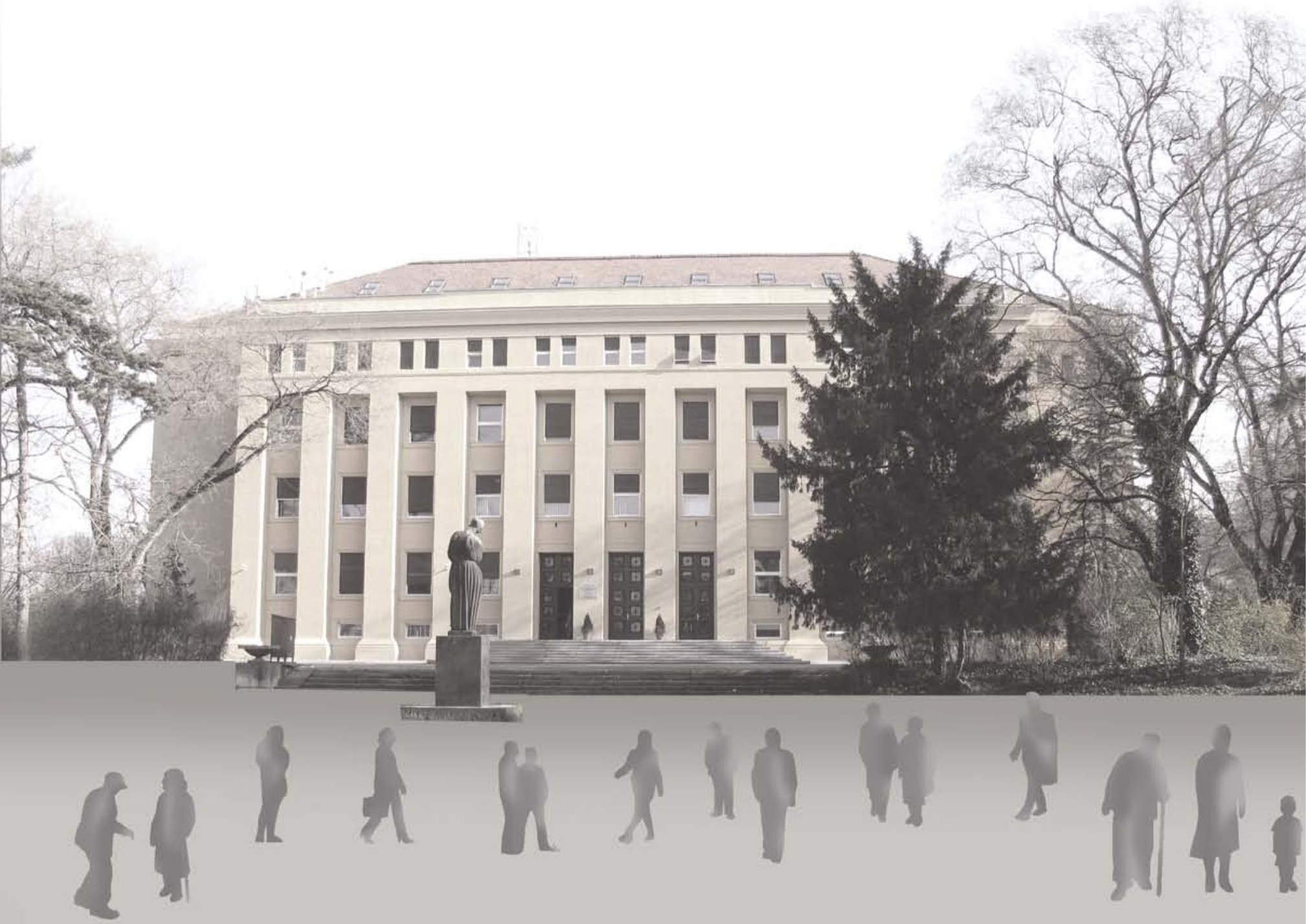





\section{A Review of Public Health Project: Zaželi - Program Zapošljavanja Žena}

Haris Ahmić1, Petra Sulić1, Laura Tomić1, Robert Likić ${ }^{2}$

${ }^{1} S$ chool of Medicine, University of Zagreb, Croatia

${ }^{2}$ Department of Internal Medicine, Unit of Clinical Pharmacology, University Hospital Centre Zagreb, School of Medicine, University of Zagreb, Croatia

Introduction and Objectives. The demographic picture of Croatia shows two trends: decreasing population and population ageing. As a consequence of these trends, the increasing social and economic burdens are placed on the National Budget. The EU-funded project „Zaželi - program zapošljavanja žena“ emerged as a temporary and partial solution for the aforementioned problems. This project with a budget of 400.900.000,00 KN created employment opportunities for crc 3000 women above 50 years of age and provided social care for around 12000 old and helpless people, located mostly in rural and remote areas of Croatia. This review aims to present the contribution of the project to women empowerment and social/health care of old people in Croatia. Methods. The review was performed using the data obtained from governmental reports on this project, Croatian Bureau of Statistics, Croatian Employment Service, as well as from the European Social Funds website. Results. Number of registered unemployed women aged 50 or more decreased by $29 \%$ in the period from 2017 to 2019. This percentage also pertains to areas of Croatia that were not part of the project, and although this is not the sole result of this project, „Zaželi - program zapošljavanja žena" had a considerable, national impact on unemployment decrease (more than 50\%) of woman over 50 years of age. For example, in Požega city, there was a decrease from 165 to 115 unemployed women, and in the city of Karlovac, the number was halved (from 349 to 172). All women employed, most having just primary or secondary education, were provided with additional education and different kinds of training: "gerontodomaćica", cooking classes, driver's license, first aid courses, occupational safety and health courses, basic computer skills and IT literacy etc. Conclusion. "Zaželi - program zapošljavanja žena“ project had a significant impact on the decline in the unemployment rate of women older than 50 years of age in Croatia. Additional education and training made women more competent and skilled which could also help them in finding future employment. Finally, more than 10000 elderly people in need received social, physical as well as psychological care.

\section{Integration of The Elderly Day Life with The Children Day Care - Example from Israel}

\author{
Adi Ashkenazi \\ School of Medicine, University of Zagreb, Croatia
}

Introduction. In our time, loneliness and boredom are common problems among the elderly age group. Elderly living alone, or in nursing homes, becoming more isolated from the younger society. This by itself, may lead to a reduction in communication cycles, lack of physical or emotional contact, and negative feelings such as uselessness, emptiness, and sadness. As a result, outcomes such as mental damage, cognitive depression and, psychological damage may develop. Aim. Present novelty idea which can ameliorate lifequality and, and decrease the risk of elderly depression and/or causative mental regression associated with loneliness and boredom. Case report. In Israel, from 2010 , dozens of private projects are being operated, to give an ideal situation for two marginal age groups, the pediatric and elderly. This is done by merging nursery homes with kindergartens. Meaning, allocating kindergartens to work inside nursing homes. Moreover, to make it even more practical, the kindergarten schedule includes playtime and care with the elderly, giving each participant the option to be an active part of this new community which is being created. Discussion. With the global development of new norms, which productivity and functionality are strong applicators of life, we have created a problem that disrupts the healthy community values. The idea of uniting the two extremity age groups is logical and yet, not that common. Inter-connecting between the elderly and children creates a new environment, where each participant benefit. To clarify, children are more likely to develop empathy, maturity, social awareness and at the same time, enjoy genuine attention. Likewise, the elderly are surrounded by an energetic environment and lively attention, with an increasing feeling of value, importance and, functionality. Conclusion. Elderly loneliness is a common problem which, should be approached and treated. A solution like presented above is one of many, which is for the benefit of the elderly and, our society's mentality and values. 


\section{The Effect of Different Exercises on Osteoporotic Bone}

Hana Hajsok, Tomislav Kottek, Costa Shani, Ivana Karla Franić, Ana Giljanović, Luka Matanović, Damir Mišura

Student society for orthopedics and traumatology, School of Medicine, University of Zagreb, Zagreb, Croatia

Introduction. Osteoporosis represents a major health problem worldwide with a rise in the number of osteoporotic fractures, expecially vertebral. Diagnose is made by dual energy $\mathrm{X}$-ray absorptiometry showing T-scores of less than -2.5 . Prevention is the strategy to avoid future potential fractures in women with osteoporosis, who have already sustained a fracture. As non-pharmacological intervention, physical activity has been proven to play a major role in the increasing of lumbar spine bone mineral density (BMD). The aim of this study, was to determine which exercises can improve bone health in adult women. Description. Mechanical load exercises produce mechanical bone stress and activation of osteoblasts. Not all exercise modalities are equally osteogenic. There are certain training programs, that are classified as appropriate exercises for adult women, diagnosed with osteoporosis. Evidence shows that weight-bearing and musclestrengthening exercises can improve; agility, muscle strength, bone strength, posture and balance. Consequently, reducing the risk of falls and fractures. Conclusion. At any stage of osteoporosis, exercise is equally important. Prolonged aerobic training showed significantly low impact on bones, lesser osteogenic response and no influence on walking speed. High-impact weight-bearing exercises (jogging, dancing, jumping), static weight-bearing exercises (single-leg standing) and high-impact non-weight-bearing exercises (progressive resistance exercise with free weights, medicine balls and elastic bands) showed improvement in muscle strength, balance and reduced fear of falling. Combination or single use of resistance training and weight-bearing exercises, prevents major bone loss after menopause. Brisk walking exercises in elderly women found improvements in postural stability. Adding a weight vest improved balance more than walking without a vest. Several case reports describe the benefit of yoga practice, which may improve bone density, but if performed incorrectly, can increase the risk of vertebral fracture. Patients who combined exercise with antiresorptive therapy or hormone replacement therapy, displayed significantly greater increases in the lumbar spine BMD.

\section{Can Elderly Sleep Without Any Medication?}

\author{
Nika Kelc, Lara Sever \\ School of Medicine, University of Zagreb, Croatia
}

Aim. Aim of this paper is to see how many people above the age of 65 years take sleep medications in Slovenia. Method. We observed two general practitioner offices in Slovenia. The number of patients above 65 years of age was taken. They were divided in two categories: from 65 to 74 years and above 74 years. We obsereved how many patients in each group are taking sleep medications, what were reasons for prescribing, which drugs they take, and if use increase with age. Results. First general practitioner office:They have 199 patients in the age group 65-74 years and in the group all above 74 years there are 158 people. Total number of patients at this office is 1899 , which means there is $19 \%$ of people above 65 years. Out of the people mentioned and divided in age groups, number of patients that are taking medications for sleep are, in the group $65-74$ years $83(41,71 \%)$ people and in the group all above 74 years there are $116(73,42 \%)$ people. Most patients report problems and ask for the medication themselves. The diagnosis set in this case is insomnia. Medications most commonly prescribed are Quetiapine ranging from $12.5 \mathrm{mg}$ to $25 \mathrm{mg}$, Trazodone and Mirtazepine. Less commonly they might be prescribed Diazepam ( apaurin ), Bronazepam (lexaurin) and Alprazolan (helix). In second general practitioner office they have 375 patients in a group 65-74 years and in group above 74 years there are 222 patients. Total number of patients at this office is 1853 , so there is $32 \%$ of people above 65 years. From group 65-74 years 41 (10,93\%) of people are taking sleeping medications. In group above 74 years $36(16,22 \%)$ are taking sleeping medications. As in practice above, most patients ask for the medication themselves and diagnosis is insomnia. Majority of patients are taking Sanval (zolpidem) $10 \mathrm{mg}$ or $5 \mathrm{mg}$. Conclusion. In conclusion, we can observe that a lot of eldely people require some kind of sleep medication to be able to have a normal night sleep. We can also see that the use of medication increases with age. It is always the patients who ask for the medication, because they are experiencing insomnia which is interfering with their normal functioning. It is hard to say that the use itself could be decreased, but what doctors do is to try to avoid benzodiazepins, because of their risk of addiction. 


\section{Active Ageing in Croatia from the Public Health Perspective}

Tihana Kuljiš', Roberto Mužić', Ivan Radilj', Mirjana Kujundžić Tiljak², Marjeta Majer², Iskra Alexandra Nola ${ }^{2}$

${ }^{1}$ School of Medicine, University of Zagreb, Croatia

${ }^{2}$ Andrija Štampar School of Public Health, School of Medicine, University of Zagreb, Croatia

Introduction and Objectives. Active ageing is defined as a process of optimizing opportunities for health, participation and security in order to enhance quality of life as people age. WHO categorized determinants of active ageing as economic, social, personal, behavioral, physical environment and health and social services. Our objective was to find out how health and social determinants are targeted through public health actions and interventions in Croatia. Methods. Data gathering was performed using keywords Healthy ageing, Public health interventions elderly, Public health actions elderly. Search included official Croatian sources: governmental and county websites, Croatian Institute of Public Health and Croatian Bureau of Statistics, in period of last 5 years. Results. The proportion of people ageing 65 and over is growing. In 2011 average age of the population in Croatia was 41.7 years and the share of population aged 65 and over was $17.7 \%$. The framework of healthy aging is defined by Croatian Law on health care and National strategy of health development. Public health interventions and actions for elderly in the field of health care and social services include numerous prevention programs provided by many local movements and organizations. Their active participation in the community aimed to health and social needs of elderly include in home support, digitized health care - social alarm projects, education for volunteers involved in elderly health and social care, active socialization activities; all founded by government and EU projects. Elderly targeted health action and interventions are not as numerous as the social ones, yet. The isolation of low socioeconomic households and individuals in rural areas with inability to access health and social services remain challenging. Conclusions. Although Croatia is following European trends in care for older people due to financial circumstance and governing bodies there is an inability to provide institutionalization and comprehensive model of care on the state level. Digitization in health care has enabled greater independence of elderly 'everyday life.

Changes in population's age should be followed by policy changes to ensure active and healthy ageing. This would enable increase of active and independent years for the elderly resulting in individual, as well as social and economic benefits for the society.

\section{How to Optimize the Salt Reduction Interventions Targeting Elderly Population?}

\author{
Matea Marjanović', Lea Kalajžić ${ }^{1}$, Antonia Precali', Ema Kuhar', \\ Emanuel Brađašević ', Iva Lukačević Lovrenčić ${ }^{2}$, Aleksandar Džakula \\ 'School of Medicine, University of Zagreb, Croatia \\ ${ }^{2}$ Andrija Štampar School of Public Health, School of Medicine, University of Zagreb, Croatia
}

Introduction and Objectives. Increased dietary sodium intake is a growing public health problem in developed countries and a risk factor contributing to a wide variety of non-communicable chronic diseases (NCD). Preventable NCDs contribute to higher morbidity, mortality, reduced quality of life of the elderly population and increased healthcare expenditure. Average daily salt intake in Croatia amounts to $11.6 \mathrm{~g} /$ day (World Health Organization recommendation is less than $5 \mathrm{~g} /$ day), and $19,41 \%$ of population is over the age of 65 . The specific aim is to detect key valid sources of information, possible opportunities and risks, define main criteria and framework necessary to optimize future interventions and develop large-scale national programs. Methods. We used PUB HUB policy coil as a tool which encompasses all necessary steps for policy analysis and covers all policy development steps necessary for comprehensive policy overview. As a starting position for this policy analysis we used World Health Organization recommendations, and international studies focused on the salt intake in the elderly population. Information for mapping of the needs and setting assessment were: national strategic documents, existing interventions, available project evaluations and published papers. Results. National Strategic plan for salt intake reduction after 2020 is not yet developed. A specific national program for salt intake reduction in elderly population does not exist. Few interventions for the general population have been developed, but still without noted relevant progress. Interventions in other countries exist, mostly small-scale interventions or randomized controlled trials targeting communities of elderly, e.g. in nursing homes. Some US states legally require inclusion of salt restriction in nutrition programs for the elderly in institutional settings. Conclusion. Preserving or improving health in countries with rapidly aging population poses a significant public health challenge and requires long-term planning. Elderly population is comprised of several subgroups, each of them requiring separate interventions - salt reduction is not an "one size fits all" solution. More studies are needed to properly assess the optimal approach, but this policy analysis provides recommendations on how to perform specific mapping of stakeholders, setting assessment and vulnerable groups which may be useful in future planning of large-scale interventions. 


\section{In-house Care for the Elderly in Town of Slavonski Brod Through the Projects of European Union}

\author{
Luka Maršić, Ana Magdalena Glas
}

School of Medicine, University of Zagreb, Croatia

Introduction and objectives. According to the latest census, conducted in 2011, there are 4284899 people living in Croatia. $17 \%$ are older than 65 years. $24 \%$ of people older than 65 years are leaving in single-person households. Slavonski Brod is 7th biggest town in Croatia. Slavonski Brod is 179th according to development. 59141 people are living in Slavonski Brod. 10 089 residents of Slavonski Brod are older than 65 years of age. Reduced mobility, health problems and social isolation are affecting the life of elderly people. Inhome help providers are trying to ameliorate the suffering and improve the quality of life of elderly people. We provide a detailed survey of in-home help organization in the town of Slavonski Brod. Methods. Data were collected in the public institutions. Shortened interview was conducted with all the personnel engaged in organization of the in-home care. Project documentation was obtained and analysed. Results. In Slavonski Brod public institutions providing in-home help to elderly people are Towns Society of the Red Cross, Town of Slavonski Brod. In-home help providers are tasked with organization of nutrition, doing housework, maintaining hygiene, providing care for the health and other technical chores. Towns society of the Red Cross, through the program "In home help", in 2019, took care of 193 elderly persons with health problems and low income. 9 in-home help providers provided 53963 services to the persons in need. Project of the Red Cross, "Wish-accomplish" employed 55 women from marginalizes sections of the society as inhome help workers. In 2019 they undertook 93766 hours of services. They distributed 660 packages of hygiene supplies. Town of Slavonski Brod organised the project "Employed". 62 women were employed. Women were over 50 years of age, some of them were disabled or victims of domestic violence. They cared for 285 elderly people in the wider urban area of Slavonski Brod. Conclusion. Croatia is dealing with a problem of increasing age of its citizens. Elderly people make up $17 \%$ of population. Poverty, disability and social isolation are difficulties facing a large part of the elderly. In-home help providers provide care and support to the elderly people. Through the projects of the Ministry of Demography, Family, Youth and Social Policy, Social welfare centers and the European Union, in the area of Slavonski Brod, 126 women were em- ployed and care was given to 754 people in need. 193 persons receive assistance based on impaired health and low income. 561 persons receive assistance through European Union Projects.

\section{Are Elderly Family Members a Burden on Your Family?}

Jennifer Förster, Ana Mičić

School of Medicine, University of Zagreb, Croatia

Introduction and Objectives. Even though elderly people are a valued part of society, they are usually not part of the working population; hence they may require financial, physical and medical support. That support often stems from the immediate family, pension funds, personal savings and health insurance. The aim of this study is to assess the financial and emotional implications of caring for an elderly family member on the rest of the family and whether or not it is a burden. Methods. For this study we constructed an online survey with 20 questions regarding the presence of and solutions for the potential burden that comes with supporting an elderly family member. The country of residence of the elderly person was required to complete the survey. The questions were related to the following: living with or financially supporting an elderly person, assessment of mental faculty and mental and physical disability, emotional impact on the caretaker, health care access and coverage of the elderly person and opinion on who should carry the burden of the ageing population. We reached the participants through social media platforms and used the snowball effect to reach more working people (parents, superiors, professors and friends). Results. Two thirds of participants do not consider taking care of their elderly family member to be a burden. One fifth are financially supporting an elderly person. Half consider financially supporting an elderly person to be some kind of a burden. Also half find the elderly persons' physical disability burdensome. One quarter of participants consider an elderly persons' memory impairment to be a burden. Two thirds experienced some emotional stress to varying degrees due to caring for an elderly person. Most elderly persons have basic health insurance, which in more than half of the cases does not cover home visits and nursing care. Two thirds attribute the majority of costs to the daily expenses, followed by medications and health care of the elderly person. Half the participants believe that society should spend money on the elderly, while the other half believe the money should be spent on children. Half believe that the government should carry the bur- 
den of the ageing population. Conclusions. Opinions on whether caring for an elderly family member is burdensome are divided and differ between countries. However, many participants agreed that the government should take responsibility for their elderly. There are participants that consider the financial aspect of caring for an elderly person significant. Even more find it stressful caring for their elderly.

\section{Vertigo in the Elderly}

\author{
Mislav Mokos', Lana Kovač Bilić ${ }^{2}$, Mario Bilić \\ ${ }^{1}$ School of Medicine, University of Zagreb, Croatia \\ ${ }^{2}$ University Department of ENT\&HNS, University Hospital Center Zagreb, \\ School of Medicine, University of Zagreb, Croatia
}

Vertigo in the older population is a very common symptom and a frequent reason to consult a general practitioner. Its clinical presentation is not very specific and its diagnosis and treatment are quite challenging. Dizziness in this situation is quite benign but it can have serious physical, functional and psychological consequences. One of the common consequences of vertigo is the risk of falls and fractures which has been recognized as a frequent and significant problem in the elderly which comprises multifactorial causes. The most common causes are vertigo, poor vision, poor muscle strength and coordination, medications that cause sedation and low blood pressure, poor sensation and impaired proprioception. One of the most common causes of vertigo is Benign Paroxysmal Positional Vertigo, but also neurologic problems, vestibular areflexia, vascular disorder and Meniere disease should be considered. Complete vestibular examination should be performed to be able to confirm the diagnosis and treat the patient. Importantly, standard caloric tests, using video systems, may show slower responsiveness, possibly due to aging issues which is important to take into consideration and individually adapt all examinations. In conclusion, a careful medical history, adequate diagnostic tools and appropriate, early and usually multidisciplinary treatment are necessary to prevent falls and fractures which can seriously affect the quality of life in the elderly.

\section{Elderly and Students Housing}

Amit Moses

School of Medicine, University of Zagreb, Croatia

Abstract. In the world of today, personal development and social efficiency are on the top priorities, and the world became nonfriendly towards the weak classes of society which usually left alone. Who is included in these groups? The great majorities are elderly, but not only, handicap, patients with disabilities and chronic diseases can be also included. It might be because they are a burden on society and they cannot contribute to the fast-developing world of today? This concerning trend became more common in developed countries leading to a silent crisis of abandoning our elderly, so what can we do about it? Introduction. While the negative demographics of Croatia is pounding and ratios between seniors to youngsters are increasing, the elder generation is left alone. The elderly are suffering from loneliness, sadness, and sorrow which have several consequences on the society, health care systems and economy. Elderly who suffers from loneliness is more prone to mental and somatic illness. The phrase "I am alone, nobody needs me anymore so I prefer to die" should not be standardized in our society and it is worth getting our full attention. We should run a policy that incorporates the young generations with the old once. Usually, those gaps are created as a result of biases. We can bridge upon those biases with a rewording system which can contribute to both sides. Aim. Our aim is to decrease the time the elderly spend alone by incorporating students in their lives and simultaneously helping students with their expenses. Case report. Similar to a project in Israel, in which students will live in the homes of senior citizens. Students who participate in the project will receive free living as well as a scholarship at the end of the year. In addition, this is a worthwhile project that connects the younger generation to the older generations. The project addresses the most burning needs of both populations. From one side the phenomenon of loneliness is common among veteran citizens living on their own, and on the other, the high housing prices with which students, mostly living on rent, have to deal. Besides, financial assistance the students will get credit points for their future residencies. Discussion. Our society is changing rapidly, values such as respect for the elderly is disappearing. Because of it, a large cut of the population is paying the price and neglected on a daily bases. Incentives such as scholarships, credit points and discounts in rent, might be a great solution for both sides. It is likely that at first, it will hard to convince both sides to cooperate, but with proper rewarding. 


\section{Cognitive Impairment and P300 Changes in Patients with Multiple Sclerosis}

\author{
Hanna Pašić', Luka Vujević1, Tea Mikula', \\ Marija Bošnjak Pašićc , Ivan Mikula ${ }^{3}$ \\ 'School of Medicine, University of Zagreb, Croatia \\ ${ }^{2}$ Department of Neurology, University Hospital Centre Zagreb, School of Medicine, \\ University of Zagreb, Croatia \\ ${ }^{3}$ Special Hospital "St. Catherine", Zagreb, Croatia
}

Introduction. Cognitive dysfunction is one of seldom analyzed features of demyelinating diseases, particularly multiple sclerosis. It can be shown by psychological testing and by electrophysiological analysis of event-related potentials. The most reliable electrophysiological parameters for the level of cognitive dysfunction seem to be the latencies and amplitudes of P300 waves. Materials \& Methods. Study included a group 50 patients (37female, 13male, mean age 37.5 years) with MS and the age and sex matched control group. Both groups were subjected to the auditory event-related potentials testing (auditory stimulation, oddball paradigm, 15\% target (high-pitched) and 85\% non-target (low-pitched) stimuli, total of 1024 stimuli in one run). Psychological testing was done using the California questionnaire. Quantitative variables were compared using Mann-Whitney Test. The Fisher Exact Test was used for comparing the qualitative data. Spearman's Rank correlation coefficient. Aim of Study. The aim was to determine the incidence of cognitive impairment among the patients suffering from multiple sclerosis and to correlate the electrophysiological findings both with the level of cognitive dysfunction and with the major features of the underlying disease (age and sex of the patients, present state of the disease activity, duration of the disease, location of demyelinating lesions, leading symptoms, and concomitant diseases). Results. The results showed that the latencies of late cortical P300 waves are prolonged in the group suffering from MS, particularly for target stimuli (frontal and parietal components). The variability of amplitudes was too great to allow any statistically significant difference between the groups. Correlation between electrophysiological and psychological findings was high and statistically significant, and significant positive correlation between level of cognitive dysfunction and the major features of underlying disease. Conclusion. Electrophysiological studies measuring late cortical P300 wave latencies can be used to indicate the level of cognitive impairment in patients with MS.

\section{The Attitude and Knowledge of Medical Students Regarding Dementia}

\author{
Josip Stojić, Milan Miloševicic, , Marina Boban ${ }^{4,5}$ \\ 'School of Medicine, University of Zagreb, Croatia \\ ${ }^{2}$ Andrija Štampar School of Public Health, School of Medicine, University of Zagreb, \\ Croatia \\ ${ }^{3}$ WHO Collaborating Centre for Occupational Health, Zagreb, Croatia \\ ${ }^{4}$ Department of Neurology, University Hospital Centre Zagreb, School of Medicine, \\ University of Zagreb, Croatia \\ ${ }^{5}$ Referral Centre for Cognitive Neurology and Neurophysiology of the Croatian \\ Ministry of Health, University Hospital Centre Zagreb, School of Medicine, \\ University of Zagreb, Croatia
}

Due to aging of population and increasing number of older people with dementia, in the near future majority of doctors are likely to spend more time caring for people with dementia. Therefore, there is a pressing need worldwide to improve undergraduate medical education on dementia in order to improve skills of healthcare professionals for competent care for people with dementia in hospital and community settings. Additionally, healthcare professionals' attitudes to dementia may also contribute to unsatisfactory care of people with dementia and should be modified. The main objective of our research was to determine attitudes towards and knowledge of dementia in a sample of final year students at the School of Medicine, University of Zagreb. Students who were willing to participate were included in the study and filled an anonymous questionnaire. The paper-and-pencil questionnaire included questions on sociodemographic data (category I); overall and specific success during the study of medicine (category II.); personal interests (category III.); personal attitudes towards dementia and experiences with dementia patients (category IV); knowledge of dementia (category V); personal opinion on the representation of dementia in undergraduate curriculum (category VI) and Croatian version of "The Alzheimer's Disease Knowledge Scale" (category VII). The study was approved by the Ethics committee of School of Medicine, University of Zagreb.

At the time of submitting this abstract, 226 students completed the questionnaire (out of approximately 281 students on the final year at the School of Medicine, University of Zagreb). Statistical analysis is currently underway and preliminary results will be presented on the conference. 


\section{Effect of Autophagy Modulators on Cardiac Aging in Mouse Models Studies: A Systematic} Review

\author{
Petra Sulić ${ }^{1}$, Haris Ahmić ${ }^{1}$ Petar Maleš ${ }^{1}$, Luka Vujević1, \\ Hanna Pašići , Filip Sedlić ${ }^{2}$ \\ 'School of Medicine, University of Zagreb, Croatia \\ ${ }^{2}$ Department of Pathophysiology, University Hospital Center Zagreb, \\ School of Medicine University of Zagreb, Croatia
}

Introduction and Objectives. Progressive structural and functional impairment of the heart is among the most significant features of aging. As a consequence, the prevalence of cardiac diseases is increasing with a constantly growing elderly population. Novel interventions are needed to address this upcoming problem. Disorders of autophagy, a lysosomal degradation process, have been suggested to have an important role in aging. This review aims to provide information about the effects of recognized autophagy modulators on cardiac aging in mouse model studies, which could be useful for understanding changes in the aged human heart. Methods. This study is based on a systematic review of medical literature. Pubmed search was done on February 8, 2020, for all existing publications, using medical subject heading terms (,,aging“; „autophagy“; „,heart"; „mice“) and text words (, ,aging“; " autophagy“; , heart“; „mice“). The abstracts were limited to English-language studies. Publications were selected for inclusion if they studied the effect of autophagy on the aging heart, using genetic or external/pharmacological modulations of autophagy. Publications were excluded if they studied only the effects of inactivation of autophagy-related genes. Our search strategy yielded 21 abstracts, out of which 10 were selected for full-text screening. Results. Full-text screening showed the beneficial or potentially beneficial role of autophagy inducers. These were rapamycin, AICAR, spermidine, oleate, and high-intensity exercise stress that targeted autophagy-related molecules, mTOR, Beclin-1, ALDH2, AMPK, FOXO, and NRF2. On the other hand, fasting in old mice, caloric restriction in young mice, and palmitate showed a detrimental effect on autophagy. Conclusion. Beneficial effects of autophagy are exerted by reducing aging-induced contractile dysfunction, slowing down the aging process, and improving health. Since these studies suggest that autophagy has an important role in aging, it represents a potentially good target for reducing age-related cardiac dysfunction.

\section{Medical Literacy and Opinion of Women on Hormone Replacement Therapy in Menopause}

\author{
Paula Pavlek², Lucija Skalicki , Zrinka Šakić1 \\ ${ }^{1}$ Health Center Zagreb Istok, Croatia \\ ${ }^{2}$ School of Medicine, University of Zagreb, Croatia
}

Introduction. Menopausal women are encountered to a greater extent with decisions about hormone replacement therapy (HRT) in the context of contradictory views and inconclusive evidence. HRT use is a health-related decision which requires detailed information, consideration of various potential risks, benefits and a woman's choice. The main reasons behind not intending to take HRT are general preference not to take medication and a belief that treatment of menopause, which is not considered an illness unless severe symptoms were present, is unnatural and unnecessary. Besides, potential side effects, fear about cancer and long-term safety concerns of HRT were one of the motives frequently mentioned in the literature so far. Objective. We aimed to evaluate whether middle-age women are prone to take HRT, their attitudes and perceptions towards using HRT, as well as specific concerns and information sources on HRT. Methods. The study was carried out during three days in February 2020 and has taken place in three family physician offices and one gynecology office in Zagreb. The anonymous survey included 9 questions and tested perspective and knowledge of participants about the benefits and risks of HRT. The participants were women $\geq 45$ years of age. Results. A total of 36 questionnaire forms were filled and used for statistical analysis. The majority of women were informed (67\%) about HRT. The leading source of information about HRT was their gynecologist (31\%). The main reasons for participants to avoid HRT (89\%) were not feeling the need to use it and the belief that menopause is a natural process that does not need to be treated. Conversely, the supporters of HRT use $(11 \%)$ cited the trust in their physician's recommendation as the main argument of approval. Conclusion: Undoubtedly, due to prejudice and insufficient education on this topic, women are unfavorably influencing their quality of life. Since nowadays the limit age of retirement and life expectancy are prolonged, the goal is to make ones' well-being optimal. As health care providers, it is important to discuss with women both the benefits and risks of HRT and make the decision depending on the debilitating symptoms they are experiencing, their medical history, family anamnesis, and supremely willingness. 


\section{Sleep Deprivation and Its Role in The Early Pathology of Alzheimer's Disease}

\author{
Laura Tomić, Petra Sulić, Haris Ahmić \\ School of Medicine, University of Zagreb, Croatia
}

Introduction and Objectives. Alzheimer's disease (AD) is the most common form of dementia and it represents one of the biggest health challenges of today's society. Sleep disturbances are one of the hallmarks of the disease, occurring long before the onset of cognitive decline. Assuming that therapeutic interventions are most efficient during the preclinical stage, the aim is to present research showing a correlation between sleep deprivation, beta-amyloid, and tau protein. Methods. To discuss the relation of sleep to the glymphatic clearance of the brain macromolecules beta-amyloid and tau, we searched PubMed with keywords sleep deprivation, Alzheimer's disease, betaamyloid, and tau. The search identified 19 studies in humans and non-human species. Only randomized control trials and review articles were included, which yielded a total of 9 results. Results. One randomized controlled study showed that one night of sleep deprivation in healthy subjects significantly increased morning beta-amyloid levels in the CSF, while the other randomized crossover study showed no significant changes in the build-up of Alzheimer's disease biomarkers. All of the review articles presented studies that show a bidirectional relationship between sleep deprivation, in terms of increased wakefulness and decreased NREM sleep, and accumulation of either betaamyloid and tau protein, or both. Conclusion. Since AD pathology occurs 15-20 years before the start of cognitive decline and given that sleep deprivation reduces the activity of the glymphatic system and therefore reduces the clearance of $\mathrm{AD}$ biomarkers, considering the important role of sleep as a treatable and a modifiable risk factor of $\mathrm{AD}$ might become a target for therapeutic intervention in the preclinical stage of the disease and a preventative strategy. Further meta-analysis should be done to estimate the significance of the studies.

\section{Attitude of Medical Students Towards Older Persons}

\author{
Luka Vujević1, Hanna Pašić ', Haris Ahmić ', Petra Sulić', \\ Branko Kolarić, $2,3,4$, Nada T. Mrčela ${ }^{2,5,6}$ \\ ${ }^{1}$ School of Medicine, University of Zagreb, Croatia \\ ${ }^{2}$ Department of Public Health Gerontology, Andrija Štampar Teaching Institute \\ of Public Health, School of Medicine, University of Zagreb, Croatia \\ ${ }^{3}$ School of Medicine, University of Rijeka, Croatia \\ ${ }^{4}$ Croatian Academy of Medical Sciences, Croatia \\ ${ }^{5}$ Alma Mater Europaea - ECM, Slovenia \\ ${ }^{6}$ Department of Health Studies, University of Split, Croatia
}

Introduction and Objectives. The progressive aging of society, caused by profound demographic changes, goes with necessity of confronting the subject of biases against the elderly. Competencies include not only knowledge and skills but also attitudes and the ability of activating and utilising them in certain situations. Medical students often get a skewed perspective on older persons, being exposed mainly to sickest ones. Education in medical school can be a part of solution to combat prejudice and improve care for older adults. The goal was to find out the attitude of medical school students in their clinical years towards older persons. Methods. Scale Test About Meaning of Your Attitude Towards Older Person by Department of Public Health Gerontology, Andrija Stampar Teaching Institute of Public Health, Zagreb, Croatia was used. Test contained 16 questions consisting of opposing descriptions of older persons. Data were processed in a way that each description had 7 possible answers (7 levels ranging from -3 to +3 ). By summation of such values results were acquired for each question. 3-day online questionnaire for students in clinical years was made where they were able to voluntarily participate. Result. 112 medical students who responded to online test were questioned in order to assess their opinions on older persons. On 10 options in the questionnaire the greatest number of participants described older persons with a score 0 , indicating that they are equally distant from both extremes and the least number of participants marked older persons with -3 , showing that the least number of participants had negative opinion of older persons in these categories. Other categories showed that regarding some traits, older persons were considered positive, with greatest number of respondents considering them useful and experienced and minority of participants regarding them opposite. On the other hand, there were some categories where greatest number of participants had such attitude, that they marked elderly as slower and more dependent on help. In the presentation, results are described in more detail. Conclusion. Most of the participants showed either positive or neutral stance towards the older persons. Many medical students still 
deem older persons as slower and more help dependent but at the same time think they are useful and experienced. More practical experience with a variety of older persons could help further improving the competences of medical students.

\section{Timing of Antiviral Therapy with Neuraminidase Inhibitors and Baloxavir for Seasonal Influenza in Older Patients}

\author{
Luka Vujević', Hanna Pašić', Emil Klarić2 \\ 'School of Medicine, University of Zagreb, Croatia \\ ${ }^{2}$ Faculty of Medicine, University of Rijeka, Croatia
}

Introduction and objectives. Seasonal influenza is an acute respiratory illness, caused by influenza viruses, which occurs in outbreaks and epidemics, mainly during winter season. Although it is usually self-limited infection (uncomplicated influenza), older individuals (of age 65 and above) are at high risk of complications, such as pneumonia and acute respiratory distress syndrome, leading to increased morbidity and mortality. Such patients are target population for treatment with antiviral drugs such as neuraminidase inhibitors (oseltamivir, zanamivir, peramivir) and the selective inhibitor of influenza cap-dependent endonuclease-baloxoavir. The aim of this study was to provide information about application and timing of these treatment options in older patients with seasonal influenza. Methods and materials. This study is based on literature review of treatments for seasonal influenza, performed in January 2020, with a focus on timing of antiviral treatment with neuraminidase inhibitors and baloxavir in older patients. Results. Antiviral therapy can shorten duration of influenza symptoms by one half to three days, with greatest benefit being associated with starting the treatment within first 24-30 hours. Also, studies suggest that early antiviral therapy reduces severity and incidence of complications of influenza, length of hospitalisation and influenza associated mortality. Despite prevailing impression that there is little or no benefit when treatment is initiated two days or more after onset of influenza, some benefit may remain for older patients even after initial 48 hours, it was also found that only $13 \%$ of patients contact their clinician within 48 hours of symptom onset. In older patients with moderate to severe symptoms, it has been found that antiviral therapy reduces symptom duration by 2.3 to 3.2 days if it is initiated 48 to 72 hours after symptom onset. Conclusion. Antiviral therapy for seasonal influenza should be ini- tiated as promptly as possible since it is most likely to provide benefit when initiated within the first 48 hours. However, therapy should not be withheld in older adults even after 48 hours of symptom onset because of proven efficacy in such patients. Nevertheless, since antiviral therapy shows greatest benefit when started earlier efforts should be directed at detecting influenza earlier in order to obtain greatest possible therapeutic benefit.

\section{A Review of Different Solutions for Automatic Fall Detection and Alerting}

\author{
Lovro Pavlaković, Goran Šeketa, Ratko Magjarević
}

Faculty of Electrical Engineering and Computing, University of Zagreb, Croatia

Accidental falls are one of the primary causes of injury among the elderly population. Prompt arrival of help is crucial for minimizing the health risks and consequences posed by such falls. Automating the fall detection process and timely alerting can greatly increase the independence and quality of life of elderly people. This has proven to be a challenging problem due to the high variety of fall events and other similar activities that a fall detection system needs to correctly classify. User comfort and privacy also need to be considered while designing such a system. Numerous solutions to this problem have been proposed and tested with high levels of success. Most of these solutions are based on measuring the acceleration of a particular part of the body of the subject using an inertial measurement unit (IMU). However, in order to increase the reliability of the detection and alerting system, multiparameter acquisition from various sensors may be used. In this paper we compare some of these solutions, including those explored at the Faculty of Electrical Engineering and Computing, University of Zagreb, and discusses potential future avenues of advancement in this field. 


\section{The Risk for OSA Increases with Advanced Age - a Large Population Study in Western Herzegovina}

\section{Sijana Demirović}

Department for Neuroscience, University of Split School of Medicine, Split, Croatia

Split Sleep Medicine Center, University of Split School of Medicine, Split, Croatia

Numerous epidemiological studies confirmed that the prevalence of obstructive sleep apnea (OSA) is varying among different age groups of the general population, with an increase in prevalence among older age groups. Since diagnostic procedures used for OSA evaluation are expensive and time consuming, appropriate assessment of the increased risk for OSA with reliable screening tools is crucial. However, the effects of age on the risk for OSA and excessive daytime sleepiness, as one of its leading symptoms, remain controversial. Thus, given the clinical and psychosocial importance of OSA and excessive daytime sleepiness, the aim of this study was to assess the risk for OSA and daytime sleepiness in a large population sample, with regard to age. A large population sample included 10108 respondents, $4748(47.05 \%)$ men and 5344 (52.95\%) women from Western Herzegovina, with the median age of 30 (IQR 20-51). The participants completed STOP questionnaire and Epworth sleepiness scale (ESS), validated screening tools for assessment of OSA risk and daytime sleepiness, respectively. An increased risk for OSA was considered as two or more positive answers to STOP questionnaire (snoring, tiredness, observed apneas and increased arterial blood pressure), while a high score on ESS (range 0-24) indicated excessive daytime sleepiness (ESS $>9$ ). Following allocation of the respondents to age groups, Chi-square test and Pearson's correlation analyses were performed. A total of 2796 (27.66\%) respondents had an increased risk for OSA, while $7312(72.34 \%)$ were not at risk for OSA. The risk for OSA increased with age $\left(\chi^{2}=1947.67\right.$, $\mathrm{P}<0.001)$. Furthermore, male respondents had a higher risk for OSA compared to female respondents in young and middle ages $\left(<21\right.$ years, $21-70$ years) $\left(\chi^{2}=11.94\right.$, $\mathrm{P}=0.018$ ). However, there was no statistically significant difference in the risk for OSA between male and female respondents in advanced age ( $>70$ years) $\left(\chi^{2}=5.678, P=0.018\right)$. Participants who had increased risk for OSA had more pronounced daytime sleepiness compared to participants who were not at risk (ESS score $7.99 \pm 4.16$ vs. $6.75 \pm 3.83, \mathrm{P}<0.001)$. However, a negative correlation was found between age of the study respondents and daytime sleepiness evaluated with ESS $(r=-0.082, \mathrm{P}<0.001$ for subjects with increased OSA risk, and $\mathrm{r}=-0.122, \mathrm{P}<0.001$ for those with no risk for OSA).The results of this study conducted on a large population sample demonstrated that the risk for OSA increases with advanced age. However, even though daytime sleepiness is one of the major OSA symptoms, it is less pronounced among subjects of older age groups, emphasizing the influence of age on OSA symptomatology. 